\title{
Efficacy of low or high-dose rituximab treatment in membranous nephropathy: a systematic review and meta-analysis
}

Naresh Kharbuja ( $\nabla$ kharbuja_8@hotmail.com )

Southeast University School of Medicine

Min Wu

Southeast University School of Medicine

Yu-Chen Han

Southeast University School of Medicine

Dan Liu

Southeast University School of Medicine

Bin Wang

Southeast University School of Medicine

Bi-Cheng Liu

Southeast University School of Medicine

\section{Research Article}

Keywords: rituximab, dose, membranous nephropathy, efficacy, meta-analysis

Posted Date: May 10th, 2021

DOl: https://doi.org/10.21203/rs.3.rs-408713/v1

License: (9) (i) This work is licensed under a Creative Commons Attribution 4.0 International License. Read Full License 


\section{Abstract}

Background: Rituximab (RTX) has emerged as a promising therapeutic option in patients with primary membranous nephropathy (MN). But the optimal dosing of RTX protocol has not been established. Recently, favorable outcomes even with low-dose of RTX has been described in MN patients. Thus, the aim of this meta-analysis is to compare the efficacy and safety between high-dose and low-dose RTX in patients with MN.

Methods: After literature search, eligible studies were further classified into high-dose and low-dose groups according to the dosage of one cycle RTX therapy. A meta-analysis was performed to evaluate remission rates and changes in biological indicators in two groups.

Results: Eight studies involving 588 patients were included in this meta-analysis. In comparison to the control groups (including cyclosporin, cyclophosphamide, chlorambucil, prednisone, nonimmunosuppressive anti-proteinuria treatment), RTX significantly improved the complete remission (CR) rate. Furthermore, there is no significant difference between high-dose and low-dose RTX in inducing total remission (TR) and CR. Also, high-dose RTX did not significantly improve serum albumin, creatine and urinary protein levels when compared with the low-dose RTX group. However, high-dose RTX did reduce the serum PLA2R antibody titers in patients. Even the difference was not significant, there was a tendency for low-dose RTX to have less serious adverse events (SAEs) than high-dose RTX groups.

Conclusion: RTX administration indicated a better efficacy than the control strategies for the treatment of primary MN. And a low-dose regimen of RTX was non-inferior to high-dose usage in inducing long-term TR up to 24 months and holds the superior tendency in preventing SAEs in MN patients.

\section{Introduction}

Primary membranous nephropathy $(\mathrm{MN})$ is one of the leading causes of nephrotic syndrome in adults [1]. Studies have shown that persistent proteinuria is the predictor of increased risk in 10-year progression to end-stage renal disease and mortality in patients with IMN. Complete remission (CR), even partial remission (PR), of proteinuria is correlated with improved long-term renal outcomes [2]. Thus, immunosuppressive strategies including corticosteroids, cytotoxic agents and calcineurin inhibitor are recommended for IMN patients in high and medium risk [3]. But a high incidence of relapse after discontinuation and substantial side-effects associated with these agents led to the search for alternative therapeutic options [4].

Recently, the role of B-cell dysfunction in the pathogenesis of $\mathrm{MN}$ has been clarified. And selective B-cell depletion of rituximab (RTX) emerges as a promising approach in patients with primary MN [5]. For instance, MENTOR trial indicated that RTX was non-inferior with cyclosporine A (CsA) in inducing CR or PR of proteinuria at 12 months, and superior in maintaining proteinuria remission up to 24 months [6]. A recent meta-analysis of 8 trials involving 542 patients showed efficacy of RTX on CR rates compared with 
the various control groups (including supportive treatment, CsA and cyclical treatment) [7]. Taken together, the accumulating evidence supported the use of RTX as induction treatment in MN patients.

Currently, the dosage of RTX administration vary widely in different medical centers. As published literatures shown, initial treatment with RTX used the conventional lymphoma protocol (4 weekly doses of $375 \mathrm{mg} / \mathrm{m}^{2}$ ), or rheumatoid arthritis regimen ( $1 \mathrm{~g}$ given twice two weeks apart), or so-called low-dose strategy (a single-dose of $375 \mathrm{mg} / \mathrm{m}^{2}$ with re-application in case of insufficient B cell depletion). However, whether lower dose of RTX is equivalent effective and safer for patients with MN is an ongoing debate. In the present study, a systemic review and meta-analysis was performed to compare the efficacy and safety of low-dose and high-dose (lymphoma and rheumatoid arthritis regimens) RTX treatment for patients with MN.

\section{Methods}

This meta-analysis followed the preferred Reporting Items for Systematic Reviews and Meta-Analyses (PRISMA) guidelines [8]. And this study has been registered in PROSPERO with registration number CRD42020198831.

\section{Data sources and search strategy}

We performed literature search in PubMed, EMBASE (OVID), WOS and the Cochrane Library database for studies published until December 31, 2020. The retrieval strategy was "rituximab", "anti-CD20 monoclonal antibodies" combined with "membranous nephropathy" or "membranous glomerulonephritis" both as medical subject headings (MeSH) and text words. Literature search was limited to article published in English but there was no restriction on race ethnicity or geographic area. The references listing of important research and review articles were manually screened.

\section{Inclusion and exclusion criteria}

The inclusion criteria were as follows: (1) randomized controlled trials, cohort studies, comparative studies; (2) adult patients (>18 year) with proven MN based on renal biopsy reports; (3) Patients treated with ACE-I or ARB for >3 months; (4) studies at least followed up for 6 months; (5) the studies published in full-length article and provided data that could be extracted from article or obtained by calculation.

Any ongoing studies, review articles, case reports, guidelines, consensus manuscripts, comments, and letters to editor were excluded. Those trials in which patients receiving a combined treatment of RTX and other immunosuppressive drugs were excluded.

\section{Data extraction and analysis}

Two independent reviewers extracted data and any disagreements were resolved by consensus. The following data were collected: the first author's name, publication year, study design, sample size, sex, age, the follow-up period, and treatment regimens. The control group is referred to patients receiving CsA, 
cyclophosphamide, chlorambucil, prednisone, non-immunosuppressive anti-proteinuria treatment (NIAT) treatment. Subsequently, studies were further classified into high-dose and low-dose groups according to the dose of one cycle of RTX therapy. The high-dose regimen included patient treated with lymphoma protocol (375mg/ $\mathrm{m}^{2}$ given 4 times at weekly intervals) or RA protocol (2 dosage of $1 \mathrm{~g} \mathrm{RTX}$ within 2 weeks). The low-dose regimen included using only one dose of RTX $375 \mathrm{mg} / \mathrm{m}^{2}$ with re-application in case of insufficient B-cell reduction or two weekly infusions at a dose of $375 \mathrm{mg} / \mathrm{m}^{2}$. Patient's serum albumin level $(\mathrm{g} / \mathrm{dl})$, serum creatinine $(\mathrm{mg} / \mathrm{dl}), 24 \mathrm{hr}$ proteinuria $(\mathrm{g} / 24 \mathrm{hr})$, PLA2R antibody depletion and adverse event were extracted from these dosage groups.

\section{Assessment of methodological quality}

Quality of each RCT studies were assessed according to Cochrane Collaboration tool for assessing "Risk of bias", and for nonrandomized trials quality were assessed based on The Newcastle-Ottawa Scale (NOS) for assessing the quality of nonrandomized studies in meta-analysis (available at http://www.ohri.ca/programs/clinical_epidemiology/oxford.asp ) [9]. NOS score of $<3,4-6$, and $>6$ indicate low-, medium- and high-quality studies, respectively. The risk of bias tool classifies as high bias, some concern and low bias for low-, medium- and high-quality studies, respectively.

\section{Outcomes}

Complete remission was defined as proteinuria of no more than $0.3 \mathrm{~g} / 24 \mathrm{hr}$ and a serum albumin level of at least $3.5 \mathrm{~g} / \mathrm{dL}$. Partial remission was defined as a reduction in proteinuria of at least $50 \%$ from baseline plus final proteinuria between $0.3 \mathrm{~g}$ and $3.5 \mathrm{~g} / 24 \mathrm{hr}$ regardless of creatine clearance or the serum albumin level. Relapse was defined as the development of proteinuria of more than $3.5 \mathrm{~g}$ after a complete or partial remission.

\section{Evaluation of the statistical analysis}

Statistical analysis of the extracted data was performed using Review Manager software (version 5.4, The Cochrane collaboration, The Nordic Cochrane Centre, Copenhagen, Denmark). Comparison of dichotomous variables were done using odds ratio (OR) and all results were reported with $95 \% \mathrm{Cl}$. For pooled OR and $95 \% \mathrm{Cl}$ estimation of dichotomous data, Mantel-Haenszel method was applied. Impact of study heterogeneity was assessed with $\mathrm{I}^{2}$ test. If the heterogeneity $\left(\mathrm{I}^{2}\right) \leq 50 \%$, then pooled OR were calculated using fixed effect model (Mantel-Haenszel), otherwise random-effect model (Der SimonianLaird) was used to calculate OR. Z-test determined the significance of OR.

\section{Results}

\subsection{Search results and trial characteristics}

Initial literature search identified 1627 relevant articles, of which 980 were from PubMed, 537 from EMBASE, 90 from Cochrane library and 20 from WOS. Using endnote software, 107 repeated studies were 
removed. 1486 studies were excluded after screening the titles and abstracts and remaining 34 articles were acquired in full-text form and 8 studies were found appropriate for inclusion in this systemic metaanalysis (Fig. 1) [6, 10-16]. The clinical and therapeutic data for 588 patients diagnosed with MN are provided by included studies. The characteristics of included studies are summarized in table 1.

\subsection{Quality assessment}

Quality of the included studies was assessed according to NOS score and Cochrane Handbook. The NOS score of these studies range from 6 to 8 and "low bias" base on the Cochrane handbook, indicating high methodological quality of included studies (as shown in Table 2).

\subsection{Efficacy of RTX in MN patients}

\subsubsection{Efficacy of RTX on TR and CR in MN}

As Fig. 2 shown, data from pooled 8 studies showed no significant difference in the efficiency between RTX and control treatment to induce TR $\left(O R=1.90,95 \% \mathrm{Cl} 0.70\right.$ to $5.16 ; \mathrm{I}^{2}$ of $82 \%$ indicating heterogenicity, $\mathrm{P}=0.21)$. However, $\mathrm{CR}$ rate in $\mathrm{RTX}$ group is significantly higher than in control group $(\mathrm{OR}=2.60,95 \% \mathrm{Cl} 1.72$ to $3.94 ; I^{2}$ of $33 \%$ indicating no heterogenicity, $P<0.0001$ ) (Fig. 3).

\subsection{Efficacy of high-dose and low-dose RTX protocols in MN patients}

\subsubsection{Efficacy of high-dose and low-dose RTX in MN patients compared with control}

Two studies reported TR with high-dose RTX. Pooled data from these studies indicated that high-dose RTX exhibited significantly higher TR $\left(\mathrm{OR}=5.49,95 \% \mathrm{Cl} 2.59\right.$ to $11.60 ; \mathrm{I}^{2}$ of $0 \%$ indicating no heterogenicity, $\mathrm{P}<0.0001$ ) than control group (Fig 4).

Similarly, two studies reported TR with low-dose RTX treatment. But pooled analysis of data revealed no significant difference between low-dose rituximab group and control group (OR=2.05, 95\% $\mathrm{Cl} 0.79$ to 5.33 ; $\mathrm{I}^{2}$ of $0 \%$ indicating no heterogenicity, $\mathrm{P}=0.14$ ) (Fig. 5).

\subsubsection{Comparison between high-dose and low-dose RTX's efficiency in MN patient}

\subsubsection{TR and CR}

Three studies compared TR and CR rates between high-dose and low-dose RTX treatment in MN. These studies totally covered 119 patients, 66 of whom were treated with high-dose RTX and 53 with low-dose RTX. As shown in Fig. 6 and 7, there is no significant difference between high dose and low dose RTX group on TR and CR. These findings suggest the overall equivalence between high dose and low dose RTX treatment groups.

After further stratification by follow-up period, we noticed that TR rate was higher in high-dose RTX group than in low-dose RTX group in those studies followed up $\leq 1$ year. But in studies followed up $>1$ year, there 
was no significant difference between high-dose and low-dose RTX groups in the TR rate (Fig. 6). The CR rates was not dramatically different between high-dose and low-dose RTX groups in studies with followup period $\leq 1$ year or $>1$ year (Fig. 7). These data implied that the longer follow-up period is needed to evaluate the response of low-dose RTX in patients with MN.

\subsubsection{Biochemical indicators}

Proteinuria (g/24hr): Two studies reported 24-hour urinary protein at the end of treatment with high dose and low dose RTX respectively. As shown in Fig. 8, the statistical analysis showed no significant difference $\left(\mathrm{MD}=0.14,95 \% \mathrm{Cl}-0.08\right.$ to $0.35 ; \mathrm{I}^{2}$ of $0 \%$ indicating no heterogenicity, $\left.p=0.22\right)$ between the two groups.

Serum albumin(g/dl): Two studies evaluated serum albumin level after different RTX regiments treatment. Pooled analysis of these data revealed that there was no significant difference between highdose and low-dose $\mathrm{RTX}$ regiment groups $\left(\mathrm{MD}=0.26,95 \% \mathrm{Cl} 0.15\right.$ to $0.37 ; \mathrm{I}^{2}$ of $0 \%$ indicating no heterogenicity, $p=0.33$ ) (Fig. 9).

Serum creatinine ( $\mathrm{mg} / \mathrm{dl})$ : Only one study assessed the serum creatine $(\mathrm{Scr})$ levels after different dosage RTX treatment ( $M D=0.60,95 \% \mathrm{Cl} 0.07$ to 1.13 ) and the result showed no significant difference between groups (Fig. 10).

PLA2R-Antibody-depleted patients: Two studies tested the serum PLA2R antibody levels in patients with IMN at end of treatment. Forty-one patients were assigned to high-dose RTX treatment group and forty patients to low-dose RTX group. The fixed- effect model was used for evaluation because of minimal heterogeneity. As shown in Fig. 11, pooled data indicated that there were more PLA2R antibody depleted patients in high-dose RTX treatment group when compared with low-dose RTX group (MD=3.20, 95\% Cl 1.09 to $9.38 ; I^{2}$ of $0 \%$ indicating no heterogenicity, $p=0.03$ ).

\subsubsection{Safety and serious adverse effects (SAE)}

Most studies reported only mild infusion reaction which could be resolved spontaneously with reducing the drug infusion rate, temporary discontinuation or with minor supportive treatment. These results suggest that both high dose and low dose RTX were well-tolerated in most patients. Here, to understand the adverse events in different RTX dose regiments with accuracy, only serious side events which required hospitalization and life-threatening (grade 3 or higher) were included. The statistical analysis showed that there was a tendency for patients receiving RTX treatment to have less SAEs than patients in the control group, but the difference was not significant (Fig.12). Only one study compared SAE between high-dose and low-dose RTX in patients with primary MN. And one patient on high-dose protocol had a severe adverse reaction. There was no such report in patients assigned to low-dose RTX treatment (Fig. 13).

\section{Discussion}


Primary MN is one common cause for nephrotic syndrome in adults worldwide[1]. Considering the detrimental effects of a prolonged treatment with glucocorticoids, steroid-sparing immunosuppressive strategies are used in patients with primary MN[17]. Recently, MN has been clearly established as an autoantibody-driven disease. Given the key role of B cells in producing pathogenic auto-antibodies, there is a clear rationale for RTX treatment in these patients $[17,18]$. During the past decade, a series of studies were performed to observe the potential of RTX as an alternative immunosuppressive therapy in primary $\mathrm{MN}[19]$. Despite the marked methodological differences limit direct comparability between studies, the overall remission rates of RTX at 12 months are ranging from $44 \%$ to $85 \%[20,21]$. And the data consistently showed the noninferiority of RTX in inducing proteinuria remission when compared with cyclosporine, steroid plus cyclical cyclophosphamide, or NIAT [11]. In the present study, we pooled the data from 8 studies and found a significant increased remission (TR and CR) in RTX group than in control group (including prednisone, cyclosporine, cyclophosphamide, chlorambucil, NIAT). These results provide further supportive evidence for the use of RTX in primary MN patients.

Although RTX is now recognized as a new first-line treatment option for $\mathrm{MN}$, its optimal initial dosingregimen is still unclear. Currently, different application protocols were used in patients with $\mathrm{MN}$ across various investigations. And the recently updated KDIGO guideline on MN proposes a wide scope of options and recommends either 2 dosage of $1 \mathrm{~g}$ RTX within 2 weeks, or $375 \mathrm{mg} / \mathrm{m}^{2}$ given $1-4$ times at weekly intervals for the initial treatment in patients at moderate or high risk for disease progression[19]. But whether lower dosage of RTX holds equivalent efficacy with more safety is an ongoing debate. Moroni et al followed patients with nephrotic syndrome and biopsy-proven primary MN for at least 1 year after receiving RTX as sole therapy. And the result showed that low-dose RTX obtained remission in $44.2 \%$ of patients [20]. A single center study performed by Bagchi et al found that $61.9 \%$ patients ahieved remission with low-dose RTX treatment [21-23]. In the present meta-analysis, we further compared the efficacy of low-dose and high-dose RTX in pooled eight clinical studies. And as the result shown, there was no significant difference between high-dose and low-dose RTX treatment in inducing TR and CR, accompanying with equivalent possibility for severe adverse effects. These findings suggest that the administration of RTX at low-dose might be a promising approach for MN with a more favorable risk/benefit profile.

Indeed, this meta-analysis has several limitations. First, small sample size of the included studies providing raw data might cause underestimation statistical bias or error. Secondly, the difference in follow-up duration among studies might cause heterogeneity in evaluating outcome. The third one is the lack of data from MN patients with reduced kidney function. Since patients with an eGFR $<30 \mathrm{ml} / \mathrm{min} / 1.73^{2}$ are rarely included in clinical trials and risk-benefit assessment, it is still unknown whether these patients could benefit from RTX treatment. Therefore, the usage of RTX in patients with progressive loss of renal function must be considered carefully.

In summary, RTX administration at either high-dose or low-dose indicated a better efficacy than the control strategies for the treatment of IMN. And a low dose regimen of RTX was non-inferior to high-dose 
usage in inducing long-term total remission up to 24 months and holds the superior tendency in preventing severe adverse effect in patient with $\mathrm{MN}$.

\section{Declarations}

\section{Acknowledgment}

The authors thank Jing Song from Southeast University School of Medicine for the editorial assistance.

\section{Statement of Ethics}

This work does not contain any study with human participants or animals.

\section{Conflict of Interest Statements}

None.

\section{Funding sources}

This study was supported by grants from the National Key Research and Development Program (2018YFC131400), Natural Science Foundation of Jiangsu Province (BK20191263) and Jiangsu Provincial Medical Youth Talent Foundation (QNRC2016817).

\section{Author Contributions}

Conception and design: N. K., M. W. and B.C. L.; data extraction: N. K. and M. W.; data analysis: N. K. and Y. C. H; Interpretation of data: D. L., B. W. and B.C. L.; manuscript writing: M. W., N. K. and B.C. L. All authors grave final approval to the manuscript.

\section{References}

1. Couser, W.G., Primary membranous nephropathy. Clinical Journal of the American Society of Nephrology, 2017. 12(6): p. 983-997.

2. Kanigicherla, D.A., et al., Long-term outcomes of persistent disease and relapse in primary membranous nephropathy. Nephrology Dialysis Transplantation, 2016. 31(12): p. 2108-2114.

3. Howman, A., et al., Immunosuppression for progressive membranous nephropathy: a UK randomised controlled trial. The Lancet, 2013. 381(9868): p. 744-751.

4. Keri, K.C., et al., Primary membranous nephropathy: comprehensive review and historical perspective. Postgraduate medical journal, 2019. 95(1119): p. 23-31.

5. Kerjaschki, D. and T.J. Neale, Molecular mechanisms of glomerular injury in rat experimental membranous nephropathy (Heymann nephritis). Journal of the American Society of Nephrology, 1996. 7(12): p. 2518-2526. 
6. Fervenza, F.C., et al., Rituximab or cyclosporine in the treatment of membranous nephropathy. New England Journal of Medicine, 2019. 381(1): p. 36-46.

7. Lu, W., et al., Efficacy and safety of rituximab in the treatment of membranous nephropathy: $A$ systematic review and meta-analysis. Medicine, 2020. 99(16).

8. Moher, D., et al., Items de referencia para publicar revisiones sistemáticas y metaanálisis: la Declaración PRISMA. Revista Española de Nutrición Humana y Dietética, 2014. 18(3): p. 172-181.

9. Wells, G. and B. Shea, O' Connell D, Peterson J, Welch V, et al. The Newcastle-Ottawa Scale (NOS) for assessing the quality of nonrandomised studies in meta-analyses. 2013.

10. Cravedi, P., et al., Efficacy and safety of rituximab second-line therapy for membranous nephropathy: a prospective, matched-cohort study. American journal of nephrology, 2011. 33(5): p. 461-468.

11. Dahan, K., et al., Rituximab for severe membranous nephropathy: a 6-month trial with extended follow-up. Journal of the American Society of Nephrology, 2017. 28(1): p. 348-358.

12. Fenoglio, R., et al., Efficacy of low or standard rituximab-based protocols and comparison to Ponticelli's regimen in membranous nephropathy. Journal of Nephrology, 2020: p. 1-7.

13. Van Den Brand, J.A., et al., Safety of rituximab compared with steroids and cyclophosphamide for idiopathic membranous nephropathy. Journal of the American Society of Nephrology, 2017. 28(9): p. 2729-2737.

14. Rosenzwajg, M., et al., B-and T-cell subpopulations in patients with severe idiopathic membranous nephropathy may predict an early response to rituximab. Kidney international, 2017. 92(1): p. 227237.

15. Seitz-Polski, B., et al., High-dose rituximab and early remission in PLA2R1-related membranous nephropathy. Clinical Journal of the American Society of Nephrology, 2019. 14(8): p. 1173-1182.

16. Cravedi, P., et al., Titrating rituximab to circulating B cells to optimize lymphocytolytic therapy in idiopathic membranous nephropathy. Clinical Journal of the American Society of Nephrology, 2007. 2(5): p. 932-937.

17. Cravedi, P., G. Remuzzi, and P. Ruggenenti, Rituximab in primary membranous nephropathy: First-line therapy, why not? Nephron Clinical Practice, 2014. 128(3-4): p. 261-269.

18. Tomas, N.M., et al., Thrombospondin type-1 domain-containing $7 A$ in idiopathic membranous nephropathy. New England Journal of Medicine, 2014. 371(24): p. 2277-2287.

19. Rojas-Rivera, J.E., S. Carriazo, and A. Ortiz, Treatment of idiopathic membranous nephropathy in adults: KDIGO 2012, cyclophosphamide and cyclosporine $A$ are out, rituximab is the new normal. Clinical kidney journal, 2019. 12(5): p. 629-638.

20. Cravedi, P., Rituximab in membranous nephropathy: not all studies are created equal. Nephron, 2017. 135(1): p. 46-50.

21. Moroni, G., et al., Low-dose rituximab is poorly effective in patients with primary membranous nephropathy. Nephrology Dialysis Transplantation, 2017. 32(10): p. 1691-1696. 
22. Ruggenenti, P., P. Cravedi, and G. Remuzzi, Rituximab for membranous nephropathy and immune disease: less might be enough. Nature Clinical Practice Nephrology, 2009. 5(2): p. 76-77.

23. Bagchi, S., et al., Low-dose Rituximab therapy in resistant idiopathic membranous nephropathy: single-center experience. Clinical kidney journal, 2018. 11(3): p. 337-341.

\section{Tables}

Tables 1-2 are available in the Supplementary Files.

\section{Figures}

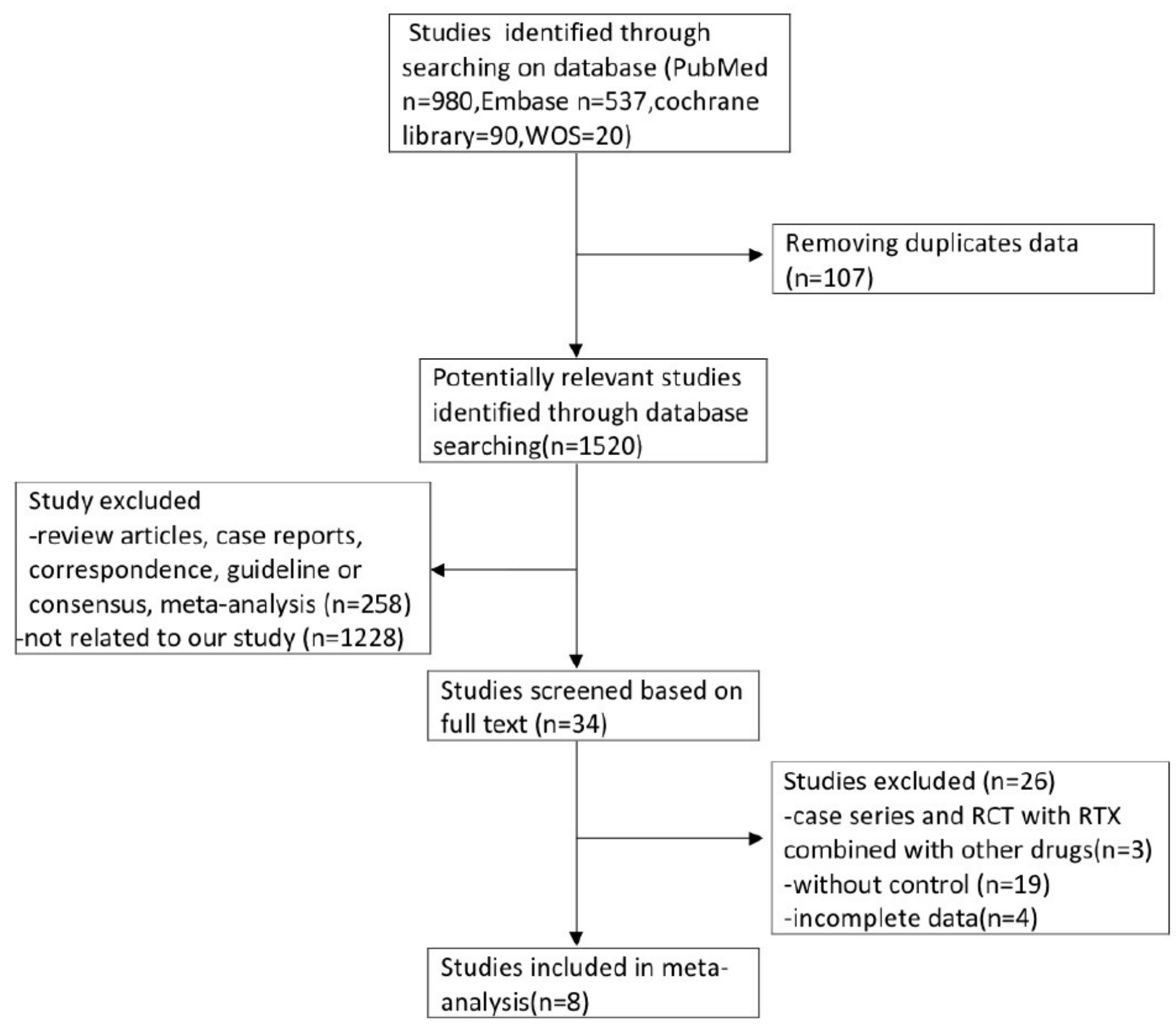

Figure 1 
Flow chart of inclusion and exclusion process.

rituximab Control Odds Ratio Study or Subgroup Events Total Events Total Weight $\mathrm{M}-\mathrm{H}, \mathrm{Random}, 95 \% \mathrm{Cl}$

\begin{tabular}{|c|c|c|c|c|c|c|}
\hline Cravedi 2007 & 8 & 12 & 16 & 24 & $12.1 \%$ & $1.00[0.23,4.35]$ \\
\hline Cravedi 2011 & 8 & 11 & 7 & 11 & $10.6 \%$ & $1.52[0.25,9.29]$ \\
\hline Dahan 2016 & 13 & 37 & 8 & 38 & $13.9 \%$ & $2.03[0.72,5.70]$ \\
\hline Fenoglio 2020 & 26 & 28 & 12 & 14 & $9.6 \%$ & $2.17[0.27,17.27]$ \\
\hline Fervenza 2019 & 39 & 65 & 13 & 65 & $14.8 \%$ & $6.00[2.74,13.15]$ \\
\hline Rosenzwajg 2017 & 9 & 16 & 2 & 9 & $10.4 \%$ & $4.50[0.70,28.79]$ \\
\hline Seitz-Polski 2019 & 18 & 28 & 8 & 27 & $13.5 \%$ & $4.28[1.38,13.25]$ \\
\hline Van den brand 2017 & 64 & 100 & 89 & 103 & $15.1 \%$ & $0.28[0.14,0.56]$ \\
\hline Total $(95 \% \mathrm{Cl})$ & & 297 & & 291 & $100.0 \%$ & $1.90[0.70,5.16]$ \\
\hline $\begin{array}{l}\text { Total events } \\
\text { Heterogeneity: } \mathrm{Tau}^{2} \\
\text { Test for overall effec }\end{array}$ & $\begin{array}{r}185 \\
9 ; \mathrm{Ch} \\
1.26\end{array}$ & & $\begin{array}{l}155 \\
\mathrm{df}=\end{array}$ & & & $82 \%$ \\
\hline
\end{tabular}

$1.52[0.25,9.29]$

$2.03[0.72,5.70]$

$2.17[0.27,17.27]$

$6.00[2.74,13.15]$

$4.50[0.70,28.79]$

$.28[1.38,13.25]$

$0.28[0.14,0.56]$

$1.90[0.70,5.16]$

Heterogeneity: $\mathrm{Tau}^{2}=1.59 ; \mathrm{Chi}^{2}=39.79, \mathrm{df}=7(\mathrm{P}<0.00001) ; \mathrm{I}^{2}=82 \%$

Test for overall effect: $Z=1.26(P=0.21)$

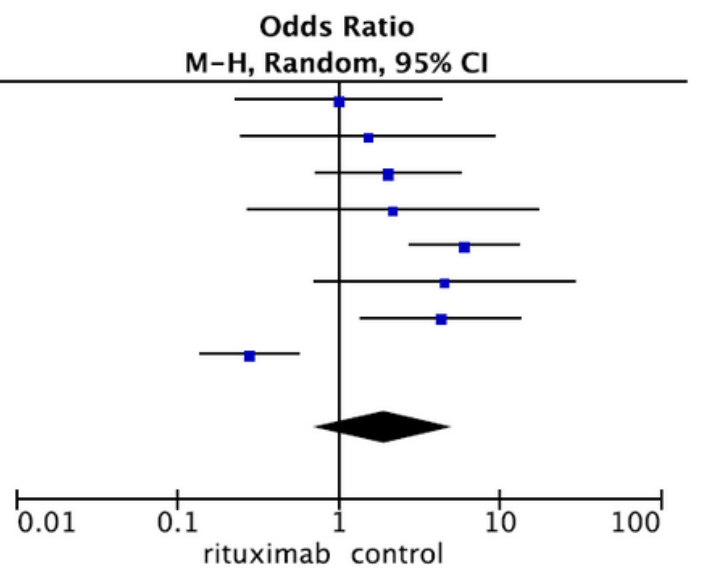

\section{Figure 2}

Forest plot shows total remission of rituximab vs control.

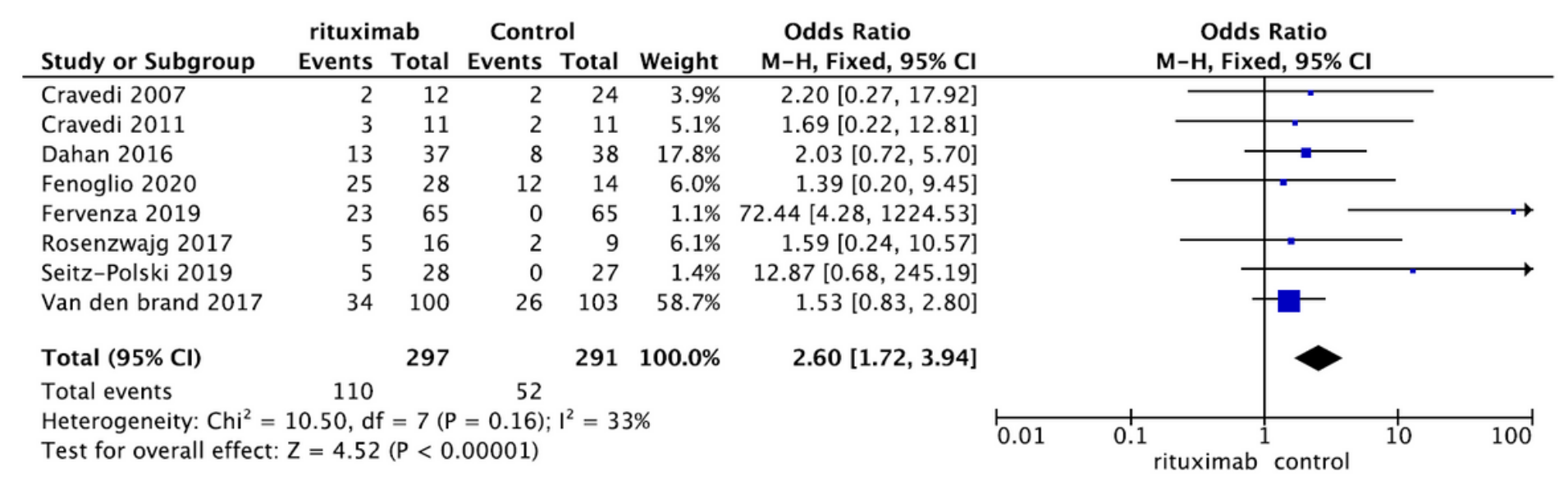

\section{Figure 3}

Forest plot shows complete remission of rituximab vs control.

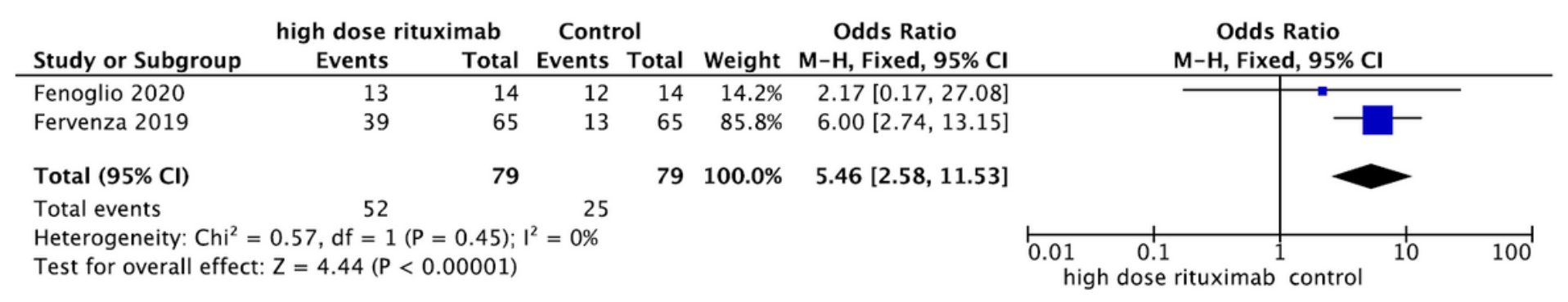

\section{Figure 4}

Forest plot shows total remission high dose rituximab vs control. 


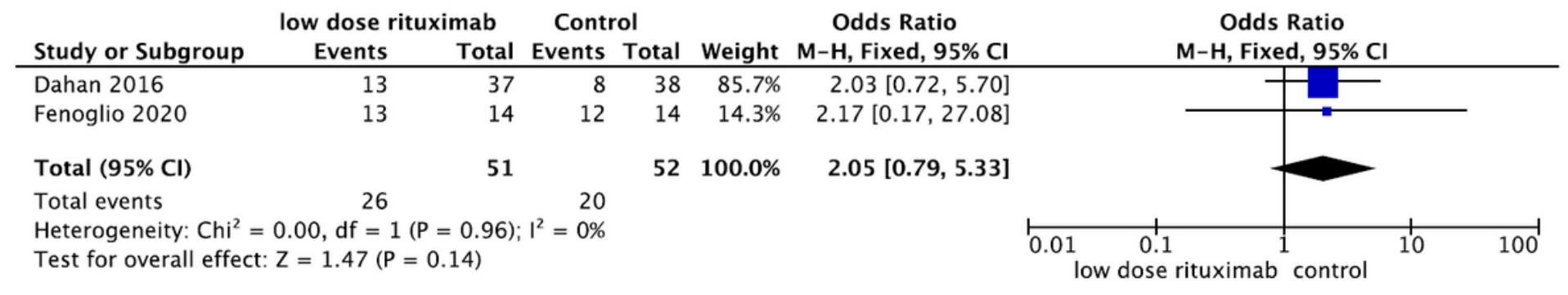

Figure 5

Forest plot shows total remission low dose rituximab vs control

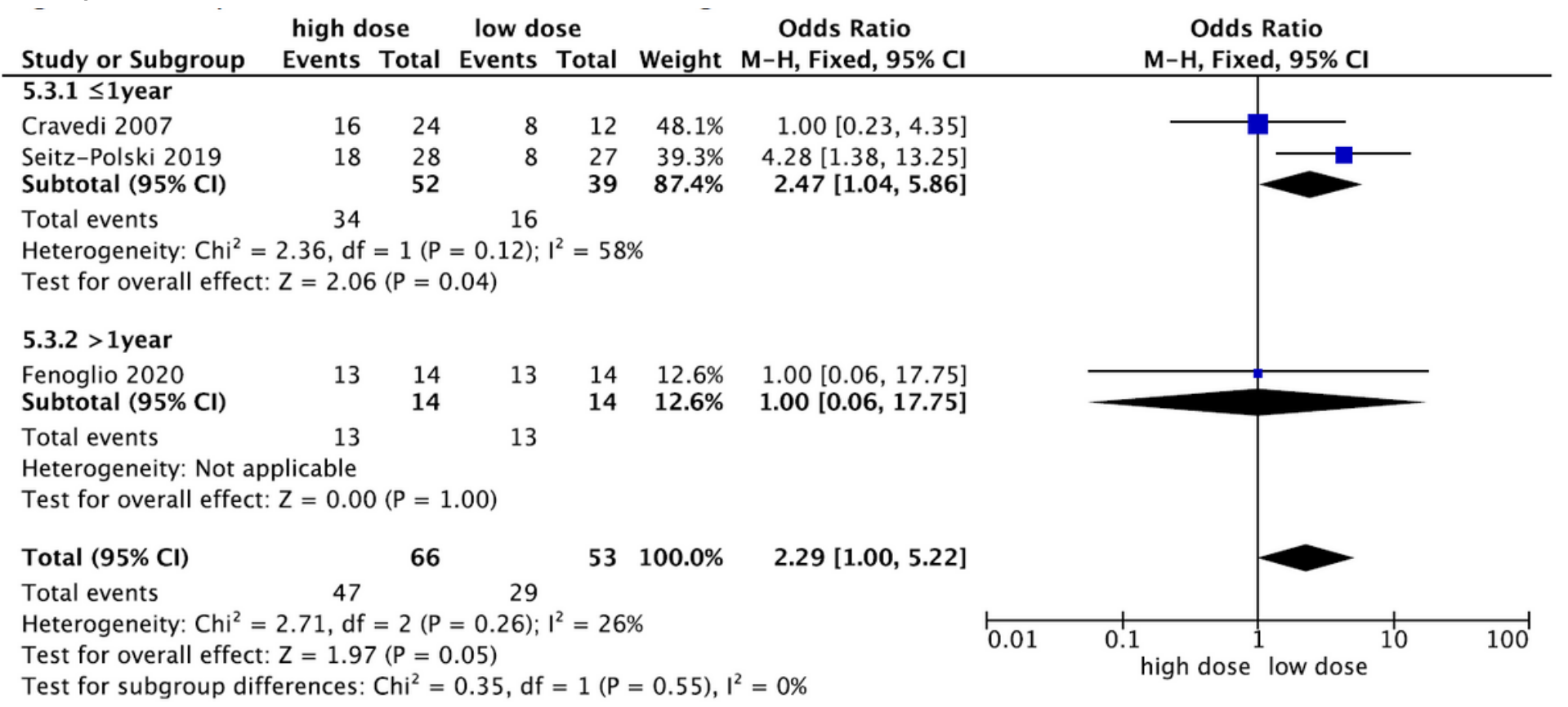

Figure 6

Forest plot shows total remission high dose vs low dose rituximab. 
high-dose low-dose Odds Ratio

Odds Ratio

Study or Subgroup Events Total Events Total Weight $\mathrm{M}-\mathrm{H}$, Random, 95\% Cl Year

4.1.1 $\leq 1$ year

Carvedi 2007

Seitz-Polski 2019

Subtotal $(95 \% \mathrm{Cl})$

$\begin{array}{lllll}2 & 24 & 2 & 12 & 39.9 \%\end{array}$

$0.45[0.06,3.70] 2007$

Total events

52

$0 \quad 27 \quad 27.3 \%$

$12.87[0.68,245.19] 2019$

$7 \quad 2$

Heterogeneity: $\mathrm{Tau}^{2}=4.39 ; \mathrm{Chi}^{2}=3.58, \mathrm{df}=1(\mathrm{P}=0.06) ; \mathrm{I}^{2}=72 \%$

Test for overall effect: $Z=0.42(P=0.67)$

4.1.2 >1year

Fenoglio 2020

Subtotal $(95 \% \mathrm{Cl})$

13

$\begin{array}{llll}14 & 12 & 14 & 32.9 \% \\ 14 & & 14 & 32.9 \%\end{array}$

$2.17[0.17,27.08] 2020$

Total events

13

12

$2.17[0.17,27.08]$

Heterogeneity: Not applicable

Test for overall effect: $Z=0.60(P=0.55)$

Total $(95 \% \mathrm{Cl})$

66

$53100.0 \%$

$1.89[0.28,12.96]$

Total events 20 14

Heterogeneity: $\mathrm{Tau}^{2}=1.28 ; \mathrm{Chi}^{2}=3.57, \mathrm{df}=2(\mathrm{P}=0.17) ; \mathrm{I}^{2}=44 \%$

Test for overall effect: $Z=0.65(P=0.52)$

Test for subgroup differences: $\mathrm{Chi}^{2}=0.00, \mathrm{df}=1(\mathrm{P}=0.98), \mathrm{I}^{2}=0 \%$

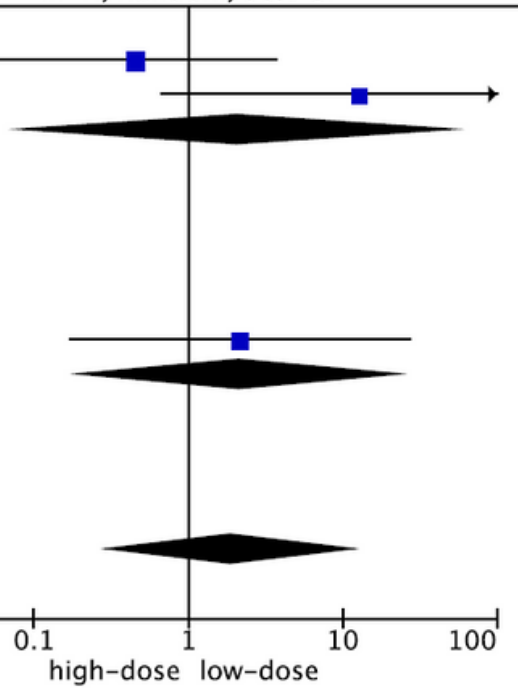

Figure 7

Forest plot shows complete remission high dose vs low dose rituximab.

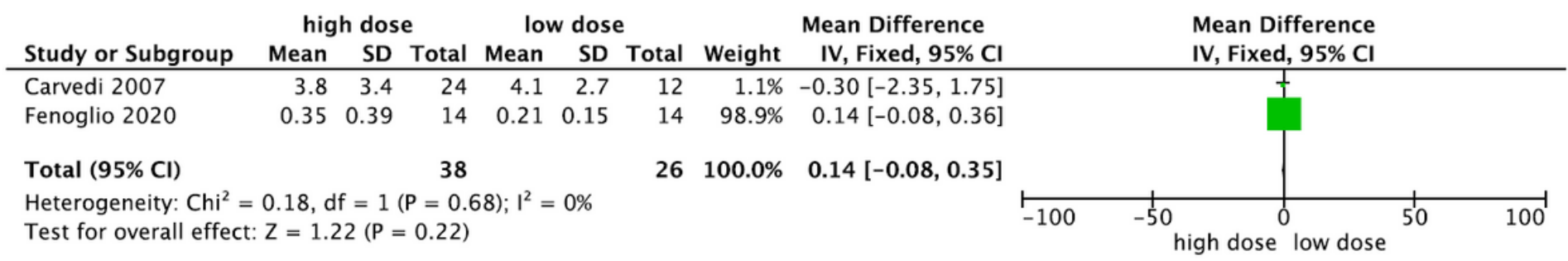

Figure 8

Forest plot shows comparison between effect of high dose and low dose rituximab for proteinuria $(\mathrm{g} / 24)$ at the end treatment.

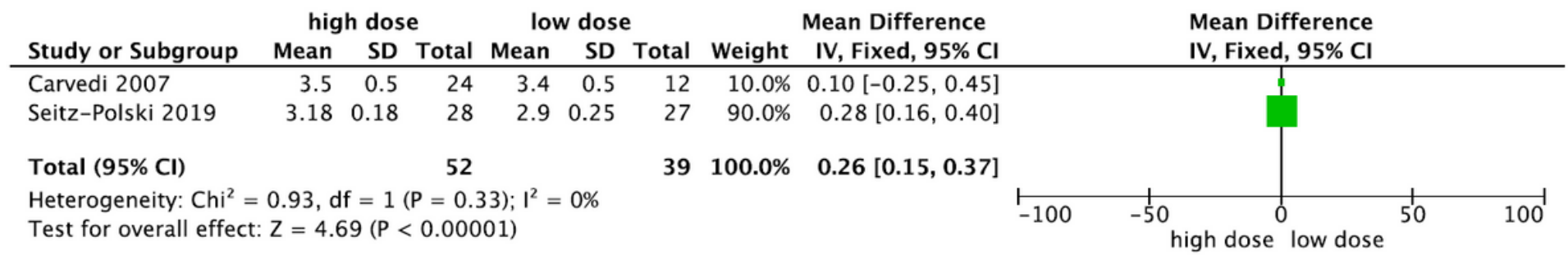

\section{Figure 9}

Forest plot shows comparison between effect of high dose and low dose rituximab for serum albumin $(\mathrm{g} / \mathrm{dl})$ at the end treatment. 


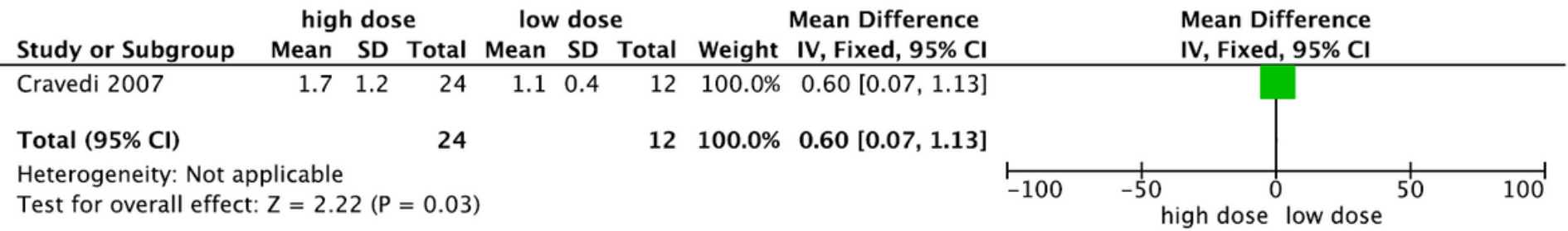

\section{Figure 10}

Forest plot shows comparison between effect of high dose and low dose rituximab for serum creatinine $(\mathrm{mg} / \mathrm{dl})$ at the end treatment.

\begin{tabular}{|c|c|c|c|c|c|c|c|c|c|c|}
\hline Study or Subgroup & \multicolumn{2}{|c|}{ high dose } & \multicolumn{2}{|c|}{ low dose } & Weight & $\begin{array}{c}\text { Odds Ratio } \\
\text { M-H, Fixed, 95\% Cl }\end{array}$ & \multicolumn{3}{|c|}{$\begin{array}{c}\text { Odds Ratio } \\
\text { M-H, Fixed, } 95 \% \mathrm{Cl}\end{array}$} & \\
\hline Fenoglio 2020 & 13 & 14 & 12 & 14 & $22.6 \%$ & $2.17[0.17,27.08]$ & & & & \\
\hline Seitz-Polski 2019 & 21 & 27 & 13 & 26 & $77.4 \%$ & $3.50[1.07,11.50]$ & & & & \\
\hline Total $(95 \% \mathrm{Cl})$ & & 41 & & 40 & $100.0 \%$ & $3.20[1.09,9.38]$ & & & & \\
\hline Total events & 34 & & 25 & & & & & & & \\
\hline $\begin{array}{l}\text { Heterogeneity: } \mathrm{Chi}^{2}= \\
\text { Test for overall effect }\end{array}$ & $\begin{array}{l}0.11, \mathrm{df} \\
\mathrm{Z}=2.12\end{array}$ & $\begin{array}{l}=1(P= \\
2(P=0\end{array}$ & $\begin{array}{l}=0.74) ; \\
.03)\end{array}$ & $1^{2}=0 \%$ & & & 0.01 & 0.1 & low dose & 100 \\
\hline
\end{tabular}

\section{Figure 11}

Forest plot shows the comparison between effect of high dose and low dose rituximab for PLA2R antibody depletion at the end treatment.

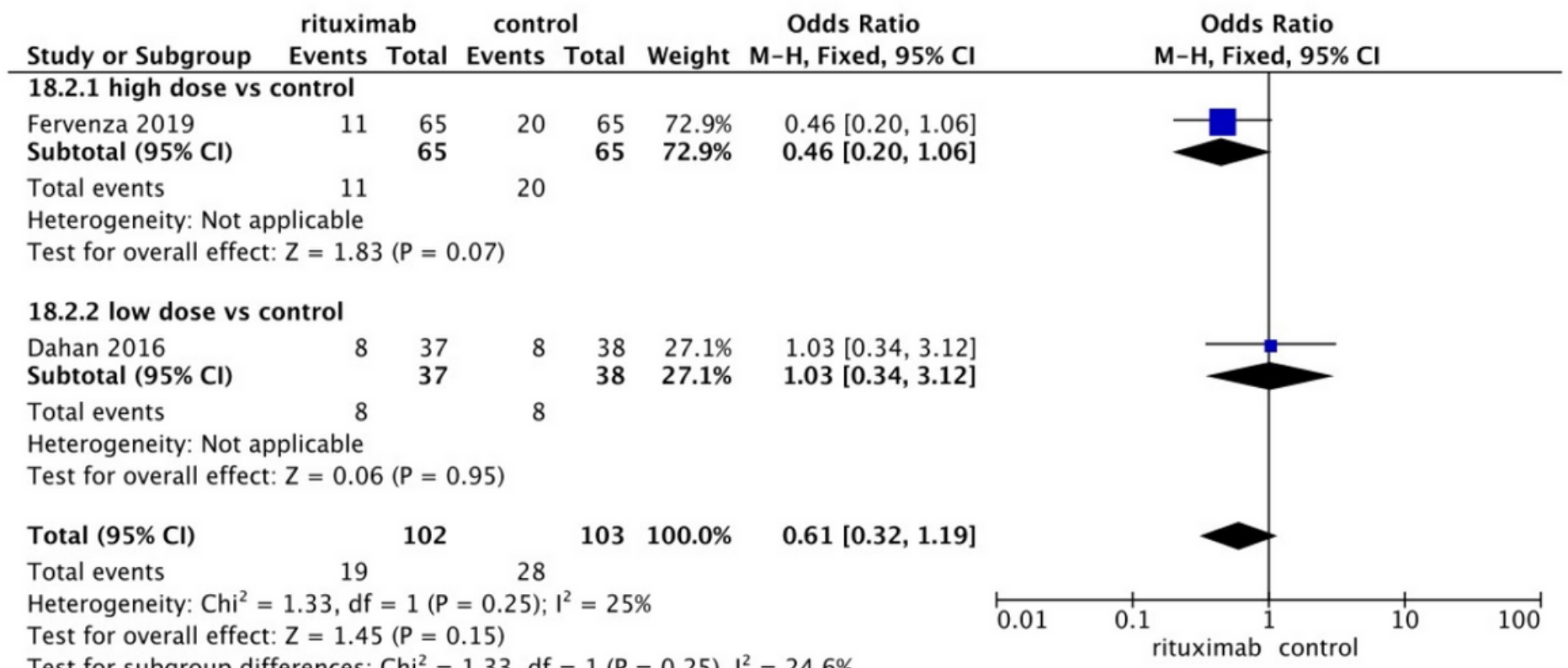

Figure 12

Forest plot shows severe adverse effect of high dose and low dose rituximab compared with control. 


\begin{tabular}{|c|c|c|c|c|c|c|c|}
\hline Study or Subgroup & \multicolumn{2}{|c|}{ high dose } & \multicolumn{2}{|c|}{ low dose } & Weight & $\begin{array}{c}\text { Odds Ratio } \\
\mathrm{M}-\mathrm{H}, \text { Random, } 95 \% \mathrm{Cl}\end{array}$ & $\begin{array}{c}\text { Odds Ratio } \\
\mathrm{M}-\mathrm{H}, \text { Random, } 95 \% \mathrm{Cl}\end{array}$ \\
\hline Cravedi 2007 & 1 & 24 & 0 & 12 & $100.0 \%$ & $1.60[0.06,42.13]$ & \\
\hline Total $(95 \% \mathrm{Cl})$ & & 24 & & 12 & $100.0 \%$ & $1.60[0.06,42.13]$ & \\
\hline Total events & 1 & & 0 & & & & \\
\hline $\begin{array}{l}\text { Heterogeneity: Not a } \\
\text { Test for overall effec }\end{array}$ & $\begin{array}{l}\text { plicable } \\
\mathrm{Z}=0.2 \varepsilon\end{array}$ & $8(P=0$ & & & & & $0.1_{\text {high dose }}^{1}$ low dose \\
\hline
\end{tabular}

Figure 13

Forest plot shows severe adverse effect of high dose vs low dose RTX.

\section{Supplementary Files}

This is a list of supplementary files associated with this preprint. Click to download.

- Tables.pdf 